\title{
An Automated Path Planning System for a Robot with a Laser Scanner for Remote Laser Cutting and Welding
}

\author{
Jens Hatwig, Pascal Minnerup, Michael F. Zaeh and Gunther Reinhart \\ Institute for Machine Tools and Industrial Management (iwb) \\ Technische Universitaet Muenchen \\ Boltzmannstraße 15, 85748 Garching, Germany \\ jens.hatwig@iwb.tum.de
}

\begin{abstract}
Remote laser beam welding (RLW) and remote laser beam cutting (RLC) are technologies that are well suited for automated applications in industrial facilities due to their contactless mode of action. The main advantages of both processes are the high operation speed, good process quality and a tailored contour of the welding seams and cutting kerfs. For an optimal use of these processes the laser beam has to be guided accurately over the work piece. A common technical realization of such processes is the combination of a laser beam deflection unit, a laser scanner and an industrial robot. This setup represents a programming challenge, as the six degrees of freedom of the robot plus the two degrees of freedom of the laser scanner lead to a kinematic redundant system. However, the advantages of this combination, the large robot workspace and the fast and precise motion of the laser scanner make it a very attractive technology for improvement of welding and cutting processes. An automated programming system for the described setup is presented in this paper.
\end{abstract}

\section{Time Optimal Robot Path - Redundant System - Combination of} Different Systems

\section{INTRODUCTION}

The implementation of remote laser processes into manufacturing promises many advantages, as the laser is a flexible, contactless and abrasion free tool. Especially the "onthe-fly" motion of an industrial robot combined with a laser scanner (see Fig. 1) can increase the process speed by a factor of 3-10 compared to resistant spot welding [1]. However, to achieve this required functionality, the path planning task has to be automated. In this paper an automatic programming system is presented. Firstly, the motivation for implementing this system is derived from the characteristics of the remote laser processes. Then, the new path planning algorithms are described in detail. Finally the description of the automated programming of the robot and the laser scanner for the production of a heat exchanger device is discussed.

\section{A. Remote Laser Beam Welding and Cutting: Process Description}

A laser beam source can be used to perform several tasks on a work piece without the need to replace or add any hardware components [2]. Only process parameters differ between welding, marking and cutting, which include the speed of the laser beam on the work piece, the diameter of the focus and the power of the laser beam amongst others. For an

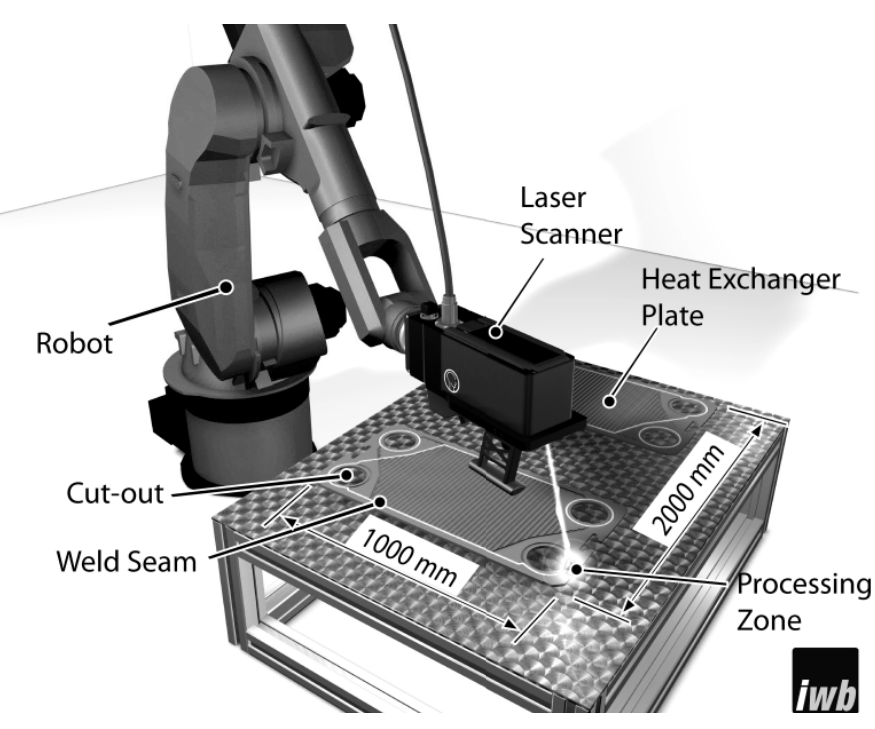

Fig. 1 Remote laser welding and cutting system with an industrial robot and a laser scanner.

adequate path planning for the robot and the laser scanner it is essential that the speed of the laser beam on the work piece remains constant which directly translates into constant process quality. This is challenging especially for an on-thefly motion. For an understanding of the path planning algorithm introduced in this work, both RLC and RLW will be briefly described.

1) Remote Laser Beam Welding: RLW is slowly becoming the main welding technology in high volume production. Daimler AG was one of the first companies that used this innovative process with a robot and laser scanner combination to improve the manufacturing of car bodies [3]. In order to call the process RLW, the distance between the laser beam forming optics and the work piece must be at least $300 \mathrm{~mm}$, but it can reach up to several meters [4]. The risk of collision is minimized by the large distance between the optics and the work piece. Moreover, the long distance enables a fast motion of the laser beam on the work piece as small movements of the robot translates to large movements of the laser beam. Hence, process speeds of about $200 \mathrm{~mm} / \mathrm{s}$ on the work piece are possible [4]. Moreover, the welding process stays stable up to inclination angles of about $\pm 20^{\circ}$ [5]. Therefore, the laser beam does not need to be perpendicularly guided over the work piece. This degree of freedom can be utilized for an optimized path planning. 
A few programming methods have been introduced to program the system with an on-the-fly motion $[3,6]$. The system described in [3] uses a master PC to synchronize the robot and the laser scanner during the process. The master PC gets the position of the robot and calculates the deflection for the mirrors of the laser scanner in the internal cycle time of the robot. In another approach, the robot path and the task for the laser scanner are generated before the process (offline) and are controlled by a dedicated external controller [6] which operates the laser scanner. The robot path is transferred to the standard robot controller unit. Both systems are already used in industrial RLW applications. A remote welding system without laser scanners is presented in [4]. The laser beam is guided over the work piece solely by the robot.

2) Remote Laser Beam Ablation Cutting: In contrast to RLW remote laser beam cutting is a new process that is not yet widely used in industrial manufacturing. However, this process has a great potential to improve the cycle time, as process speeds of up to $6,000 \mathrm{~mm} / \mathrm{s}$ are possible. With a focus diameter of $50 \mu \mathrm{m}$ the material is partly vaporized. The resulting gas pressure is used to force the molten material out of the cutting kerf. This cutting process is a layer by layer removal of the material, about 50-100 $\mu \mathrm{m}$ per exposure of the laser beam are ablated. The layer by layer removal leads to an effective cutting speed depending on the thickness of the work piece [2]. These latter two aspects represent the main differences between RLW and RLC. To implement this process the working field of the laser scanner has to be situated exactly in the same layer as the work piece. Therefore, the inclination angles are limited to the deflection of the laser scanner. A maximum of about $20^{\circ}$ is reachable.

The lack of an intuitive or automated programming of the combined system of the laser scanner and the industrial robot is one reason for the difficulties to apply this process to the industrial manufacturing [7].

\section{B. System Setup}

For reaching process speeds of more than $6,000 \mathrm{~mm} / \mathrm{s}$ the use of laser scanners is obligatory. This laser beam deflecting device consists of two mirrors to position the laser beam and several lenses to focus the laser beam on the work piece. As the mirrors have a low mass and therefore a small inertia the laser scanner can guide the laser beam faster and with a higher precision over the work piece than an industrial robot. The laser scanner has a working field of around $300 \times 300 \mathrm{~mm}^{2}$ at a distance of $400 \mathrm{~mm}$. In the project described here a laser scanner from Arges GmbH (Welding Elephant) is mounted to an industrial robot from Reis $\mathrm{GmbH} \& \mathrm{Co}$. KG (RV 60-60). In order to validate the flexibility of the programming system it was also tested with another robot (KR60 HA from KUKA $\mathrm{GmbH}$ ). The laser beam source is a $3 \mathrm{~kW}$ single mode fibre laser from IPG GmbH. Today, this laser beam source is the only one on the market which has sufficient brilliance for RLC [2]. The whole system setup is shown in Fig. 1. One main difference in the controls of both systems is the internal cycle time. The laser scanner operates at $10 \mu \mathrm{s}$, whereas industrial robots operate at more than $1 \mathrm{~ms}$.

\section{Problem Definition}

There are several commercial path planning systems for RLW that have already proved their application in industry. However, higher operation speeds and further new process parameters like multiple exposures of the cutting kerf at RLC require new path planning strategies [7].

An automated path planning is obligatory, as the combined motion of the robot and the laser scanner cannot be manually programmed at the required process speeds without adversely affecting process quality. Furthermore, the robot path should be optimized with respect to the total cycle time. The cycle time can be reduced to a minimum, if the nonproductive process time, the laser off time, is minimized [8].

Additionally, in small and medium sized enterprises an expert for programming industrial robots is not always available and the expert for the welding and cutting processes has to be able to program the cutting and welding robot. Therefore, the programming system has to be intuitively usable.

\section{GENERAL ASSUMPTIONS AND CONCEPT}

For the application of remote processes, a task oriented programming is a convenient tool to program a robot $[2,4,6$, 8]. Using this programming method the task itself is described instead of the motion of the robot. On the one hand the task description contains the geometric information of the welding seams and cutting kerfs. Normally, this is done in the robot coordinate frame to use the repeatability of the robot instead of the absolute accuracy. If the geometric information is gathered from CAD data, best fit programs can be used to position the task with respect to the robot $[6,9]$. On the other hand the process parameters are stored together with the geometric information. Once the task is defined a path planning system can generate the path for the robot and the laser scanner using the process parameters.

\section{A. General Assumptions and Simplifications}

The used system setup has a few restrictions that have to be regarded. Therefore, the following assumptions and simplifications are made.

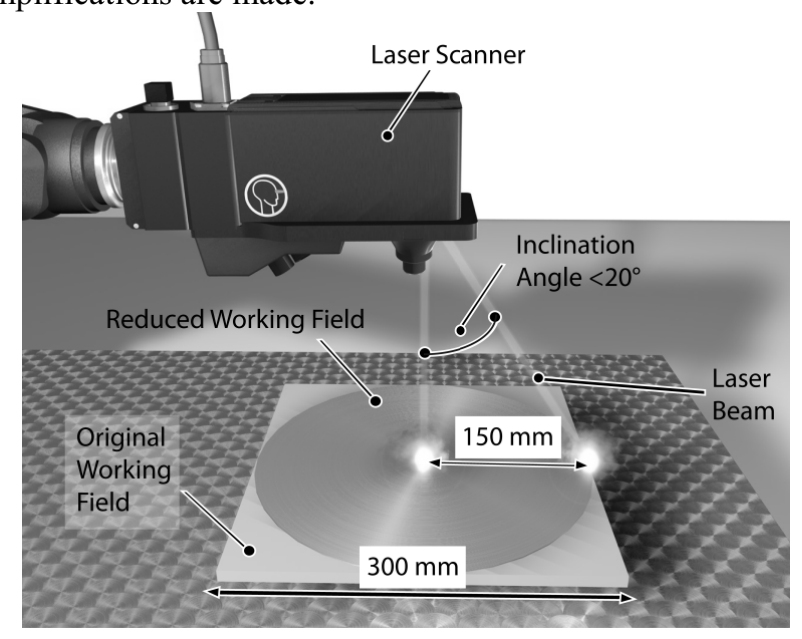

Fig. 2 Working field of the laser scanner. 
Both, the cutting kerfs and the welding seams are denoted as subtasks in this paper. However, in terms of the path planning algorithm there is no difference between them.

The working field of the laser scanner is reduced to a circle. The corners are neglected (see Fig. 2). A round working field is easier to handle, as the working field of the laser scanner can be described using only the radius of the resulting circle and the position of its center. The center of the circle is the tool center point of the robot. This can be done as the quality of the process diminishes as the inclination angles rise [4]. Therefore, the maximum inclination angles are the same in every direction. The inclination can also be limited by reducing the radius, e.g. if the accessibility is limited for some subtasks because of the clamping device.

The path of the robot has to be known in advance, as the programs of the robot and the laser scanner run synchronously on their controllers [8]. Due to the high process speeds and the required accuracy at multiple exposures with a $50 \mu \mathrm{m}$ focus diameter the robot guides the laser scanner on a straight line (LIN-movement) over the work piece while RLC is in progress. Short delays for starting the laser scanner by the robot would result only in a displacement of the whole corresponding subtask. If the robot moves on a circle or a spline curve and the start of the laser scanner is delayed, RLC cannot be performed, as the geometric shapes of both systems will not correspond to each other anymore. Additionally, the work piece and the working field of the scanner have to fit exactly. The focus of the laser beam has to remain in small ranges in the direction of the laser beam on the work piece, inside the so called Rayleigh-Length [2, 4, 5, 7]. If the mirrors deflect the laser beam, the scanner updates the focus distance so that the focus stays in one area (see Fig. 2). Therefore, the LIN-movement has to be parallel to the work piece. Thereby the complexity of the path planning is reduced to a $2 \mathrm{D}$ problem. As the robot has a 3D workspace and the work piece does not need to lie horizontally, the whole system can process $2,5 \mathrm{D}$ work pieces with the same programming strategy as long as each subtask lies solely in one planar layer. For processing subtasks that fit slightly curved layers, the laser scanner has to refocus the laser beam on the work piece. Hence, the programming strategy can be used for 3D work pieces, too, and the reduction does not eliminate any solution.

\section{B. Concept for the Path Planning Algorithm}

The calculation of the path for the robot is divided into five steps according to the concept shown in Fig. 3. The path planning for the robot (step 3) is the main challenge, as this path defines the cycle time to process the work piece.

1) First Step: The task is analyzed in order to calculate the size of the task, the number of the subtasks, the used processes and the dimension of the task. This information is used in the following steps to generate the paths for the robot and the laser scanner.

2) Second Step: Based on the characteristics of the task a suitable system setup is recommended. The accuracy can be increased by solely moving the minimum number of axes that are necessary to manufacture the work piece.

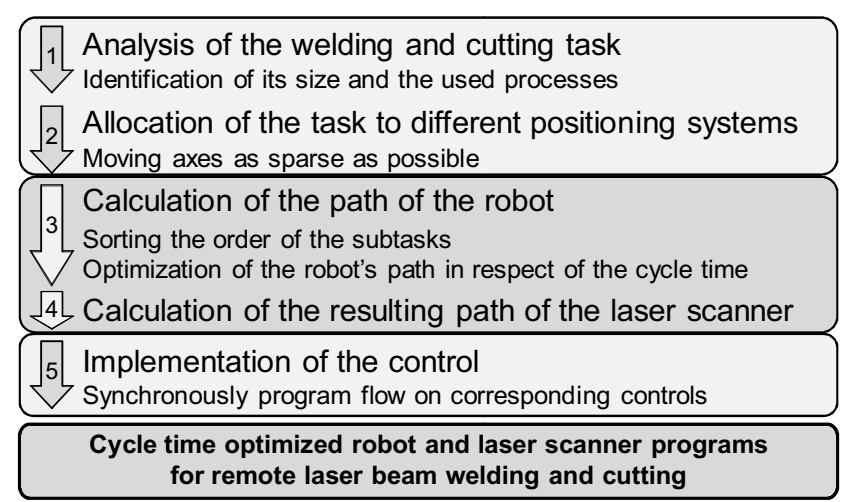

Fig. 3 Concept for the path planning for remote laser cutting.

3) Third Step: The path for the robot is generated with respect to the task and the system setup. The focus of this paper lies on the path planning for the robot, which represents the main challenge for an optimal cycle time.

4) Fourth Step: Having computed the path of the robot, the task for the laser scanner can be calculated. This is done by only using geometric transformation.

5) Fifth Step: The last step contains the implementation of the control. Two postprocessors, one for the laser scanner and one for the robot, are used to transform the paths into machine readable programs. The robot is the master system and it starts the laser scanner. Both programs start running synchronously in order to machine the work piece.

\section{New APPROACH FOR the AUtOMAted PATH PlanNing FOR REMOTE LASER BEAM CUTTING}

Using the described concept the main challenge is the path planning for the robot. A new approach to guide the robot over the work piece is presented in this chapter.

\section{A. Calculation of the Robot's Motion}

In this section the calculation of the robot's motion, which is derived of a given welding and cutting task, is described according to the presented concept. For demonstration purposes, the path planning is shown for the manufacturing of a heat exchanger device (see Fig. 1 and Fig. 4). Eight holes have to be cut into two plates, which have a dimension of about $1000 \times 500 \mathrm{~mm}^{2}$ each. Therefore, the robot has to be moved while processing the work pieces. The circular typed holes have a diameter of $120 \mathrm{~mm}$ and the thickness of the plates is $0.7 \mathrm{~mm}$. The path planning is divided into three fundamental steps.
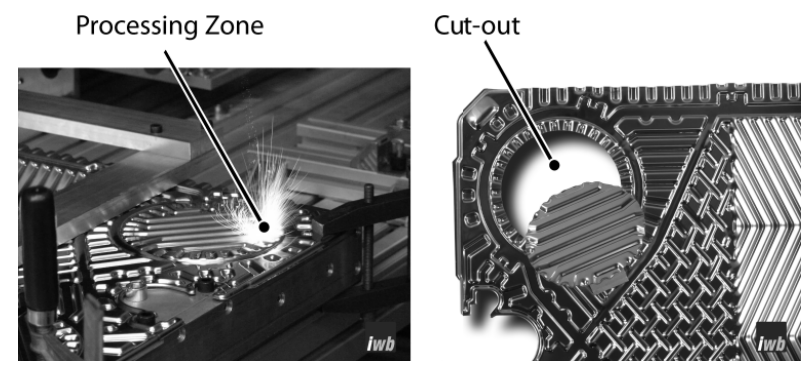

Fig 4: Cutting of a heat exchanger plate (left) and results of the cutting and welding process (right). 


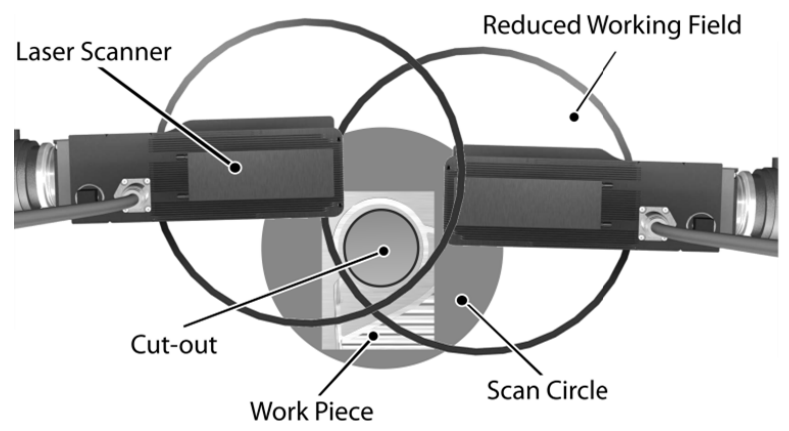

Fig. 5 Definition of the "Scan Circle".

1) Scan Circles: At first the valid positions of the laser scanner above each subtask are defined. For an analytical computation this area is described in a circular shape (see Fig. 5).

In the following, the biggest circle that fits into the intersection area will be called the "Scan Circle", as it corresponds to the area from which the laser scanner can operate. Therefore, the smallest circle, which covers the whole subtask, is calculated. The corresponding Scan Circle is derived from the position and size of the subtask and the size of the working field of the laser scanner. If the subtask is not a circle, a deviation between the Scan Circle and all valid positions occurs. Additionally, in case the size of the subtask is small compared to the working field of the laser scanner, the relative error is insignificantly small. Having the subtasks that nearly fill in the whole working field of the laser scanner, two Scan Circles for two different directions can be declared manually in the task description to compensate the deviation. This needs to be done for very specialized tasks, only.

Hence, the subtasks that have to be processed can be represented as a set of circles that the laser scanner has to cover during operation. The resulting path of the robot can be represented by a straight line through these Scan Circles.

2) Generation of the Initial Robot Path: The next task is to find straight lines in a two dimensional space that intersect with all scan circles defined in the task. The subtasks are sorted so that passing all center points of the Scan Circles leads to the shortest way (Traveling Salesman Problem).

In order to get the initial path of the robot, the start of the first straight line is placed at the center of the first Scan Circle. The direction is pointed to the middle of the second Scan Circle. With the so called End Function (this function is described in the next section) further Scan Circles are added to the straight line. If a subtask cannot be added, a new line is started. All lines are connected with a fast point-to-point movement (PTPmovement) of the robot.

For the cutting outs of the heat exchanger plates two initial lines are computed (see Fig. 6). The sections, when the laser is operating, are placed at the first possible position on the straight line. Thereby, the laser scanner has to stay inside in each Scan Circle long enough to process the corresponding subtask. The processing time $T_{\text {Subtask }}$ of each subtask can be calculated out of its length $l_{\text {Subtask }}$, its process speed $v_{\text {Process }}$ and its number of exposures $n$, according to (1).

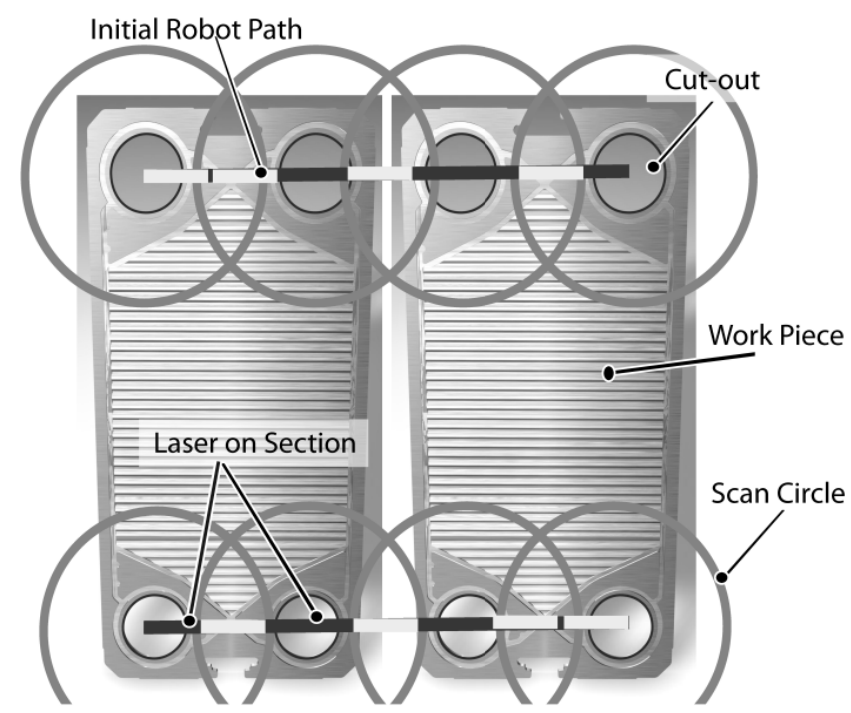

Fig. 6 Initial path through the Scan Circles of the heat exchanger plates.

$$
T_{\text {Subtask }}=\frac{l_{\text {Subtask }}}{v_{\text {Process }}} n
$$

The lengths of these "laser on" sections are defined by $T_{\text {Subtask }}$ and the speed of the robot $v_{R o b o t}(2)$.

$$
l_{\text {Section }}=v_{\text {Robot }} \cdot T_{\text {Subtask }}
$$

As $T_{\text {Subtask }}$ is given by the process and is therefore not changeable, $l_{\text {section }}$ can be varied only by $v_{\text {Robot }}$. An adjustment takes place, which ensures that every subtask can be processed on the initial robot's path. Experiments showed that $v_{\text {Robot }}$ cannot exceed $200 \mathrm{~mm} / \mathrm{s}$ for RLC, as higher speeds reduce the kerf quality until the process breaks down, due to high vibrations of the robot.

With this initial robot path the work piece can be processed, if the task for the scanner is calculated and transferred to the controllers according to step four and five of the concept. The cycle time for the whole process, however, is not considered, yet.

3) Path Optimization: The optimization of the cycle time is only demonstrated for the first four subtasks. Shortening the path of the robot leads to the shortest cycle time for the whole task, as the process time cannot be changed. For a better visualization of the method the subtasks have been displaced from one line, as shown in Fig. 7. This makes the problem more difficult to solve. Calculating the path for the heat exchanger plates is easier, as all the subtasks lie on one straight line.

For the algorithm presented in this paper it is more convenient to use the direction of the robot path in a rotated coordinate frame. Thereby the y direction points in the same direction as the straight line of the robot. This is possible, as the whole computation process is repeated for several directions. Therefore, in each repetition the direction is known. The corresponding $\mathrm{x}$ value in this coordinate frame is called offset. The path of the robot is defined by its start and end point, only. Now, the laser on sections can be defined by their start points (y value) and $l_{\text {section. }}$. 


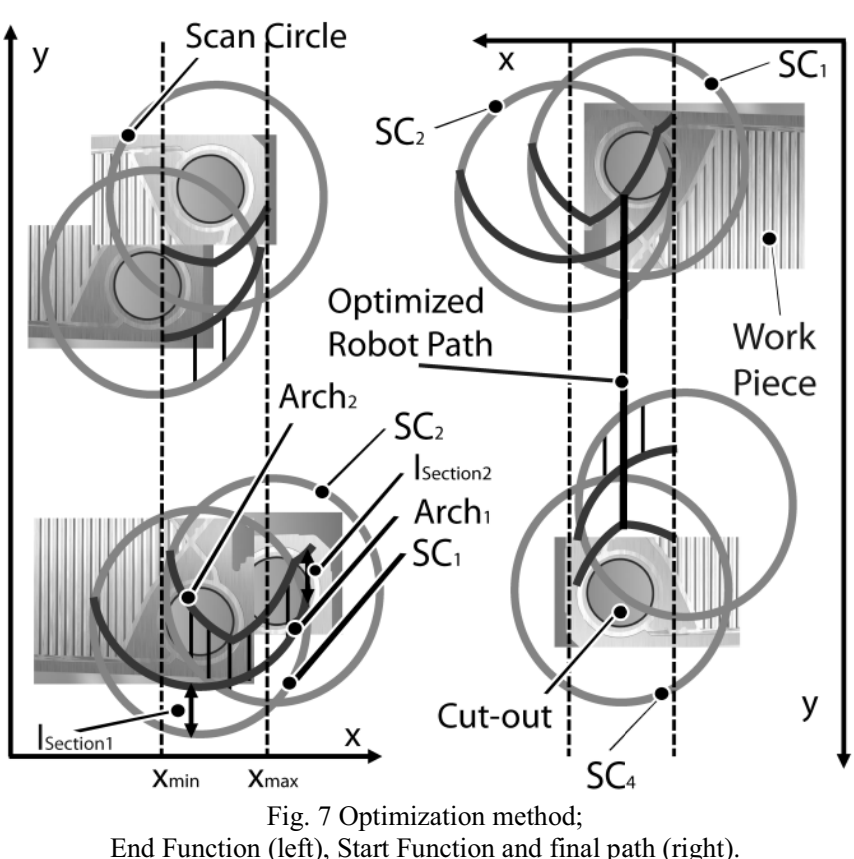

To optimize the length of the robot path and the positions of the sections on it three functions are necessary: The End Function (EF), the Start Function (SF) and the Length Function (LF).

The EF defines the first possible end of the straight line for each offset. First possible end means the lowest possible y value. The corresponding SF tells the last possible start of the straight line that allows processing all subtasks before reaching the $\mathrm{y}$ value of the $\mathrm{EF}$. Using the $\mathrm{SF}$ and the $\mathrm{EF}$, one can compute the Length Function. It yields for each possible offset the length of the resulting robot path.

The calculation of EF is shown in Fig. 7 on the left side. When starting as early as possible, the first Scan Circle $\left(\mathrm{SF}_{1}\right)$ can be finished at the first $\operatorname{arch}\left(\operatorname{Arch}_{1}\right)$. The distance between the $\mathrm{SC}_{1}$ and the $\mathrm{Arch}_{1}$ is $1_{\text {Section1 }}$. The possible offset is the whole width of $\mathrm{SC}_{1}$, as $\mathrm{Arch}_{1}$ depends on $\mathrm{SC}_{1}$, only. The precondition for this operation is an arch that cannot interact with $\mathrm{SC}_{1}(3)$.

$$
\operatorname{Arch}_{0}=-\infty
$$

The second arch $\left(\mathrm{Arch}_{2}\right)$ depends on $\mathrm{SC}_{2}$ and $\mathrm{Arch}_{1}$. It shows the first possible points in which processing the second subtask can be completed. In general, $\mathrm{Arch}_{\mathrm{i}}$ that shows the points after processing subtask i can be computed as:

$$
\begin{aligned}
& \operatorname{SCBW}_{i}(x)=\max \left(\operatorname{Arch}_{i-1}(x) ; \operatorname{ISC}_{i}(x)+l_{i}\right) \\
& \operatorname{Arch}_{i}(x)=\left\{\begin{array}{l}
S C B W_{i}(x), \text { if } S C B W_{i}(x)<u S C_{i}(x) \\
\text { undefined, else }
\end{array}\right.
\end{aligned}
$$

In (4) $l S C_{i}$ is the lower half of the Scan Circle, $u S C_{i}$ is the upper half and $l_{i}$ is the length of the processing section of subtask i. The End Function is the last Arch (5), which is defined for the valid offsets.

$$
E F(x)=\operatorname{Arch}_{i_{\max }}(x), x \in\left(x_{\min }, x_{\max }\right)
$$

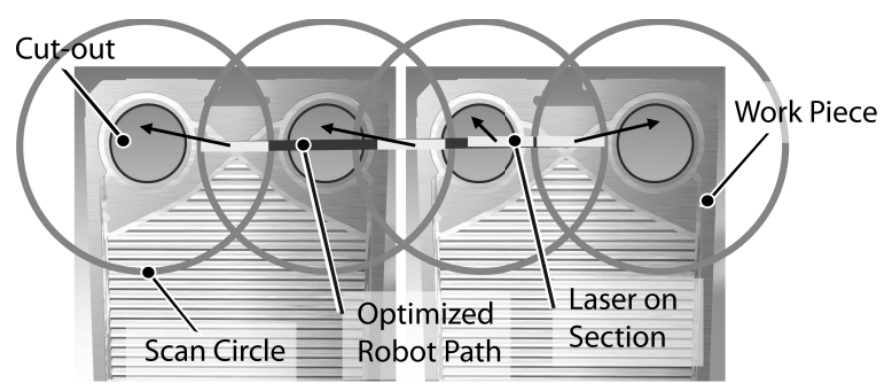

Fig. 8 Cycle time optimal path through the scan circles.

The calculation of EF is shown in Fig. 7 on the left side. When starting as early as possible, the first Scan Circle $\left(\mathrm{SF}_{1}\right)$ can be finished at the first arch $\left(\mathrm{Arch}_{1}\right)$. The distance between the $\mathrm{SC}_{1}$ and the $\mathrm{Arch}_{1}$ is $1_{\text {Section } 1}$. The possible offset is the whole width of $\mathrm{SC}_{1}$, as $\mathrm{Arch}_{1}$ depends on $\mathrm{SC}_{1}$, only. The precondition for this operation is an arch that cannot interact with $\mathrm{SC}_{1}$ (3).

After knowing EF and SF one can compute LF as shown in (6). It is piecewise differentiable because it has been created by adding or subtracting piecewise differentiable functions.

$$
\text { LengthFunction }(x)=E F(x)-S F(x)
$$

Therefore, the minimum of $\operatorname{LF}(\mathrm{x})$ can be computed, which is the optimal offset. In Fig. 7 this is shown by the Optimized Robot Path.

Using this optimal offset, the final straight line is created. The straight line starts at $\mathrm{SF}$ (offset) and finishes at $\mathrm{EF}$ (offset) in the rotated coordinate frame of Fig. 7. To get the straight line in the coordinate frame of Fig. 6, it is rotated according to the initially specified direction.

After all previous calculations, there is only one necessary value left: the direction. In this implementation systematic sampling is utilized. Systematically sampling in this context means to try a number of equally distributed values for the direction and use the best result created by this method. Having calculated the best direction the $2 \mathrm{D}$ path is transformed to the 3D path used for the actual robot. The final path for the robot is shown is Fig. 8.

\section{B. Calculation of the resulting path of the laser scanner}

As soon as the optimal robot path is known, the task for the scanner can be easily calculated using a simple geometrical transformation. As shown in Fig. 9, due to the complex movement, it is obvious that manual online programming of the two devices is very complicated if not outright impossible [8].

\section{Implementation of the Control}

A standard industrial robot and a standard laser scanner are used for implementing RLC and RLW. As no extra hardware and hence no extra programming effort are needed, the costs are reduced compared to $[3,6]$.

The controllers of the robot and the laser scanner can read ASCII coded programs in their respective programming language. Therefore, the calculated path of the robot is transformed into a robot program via a postprocessor and sent to the controller. The same is done for the tasks of the laser 


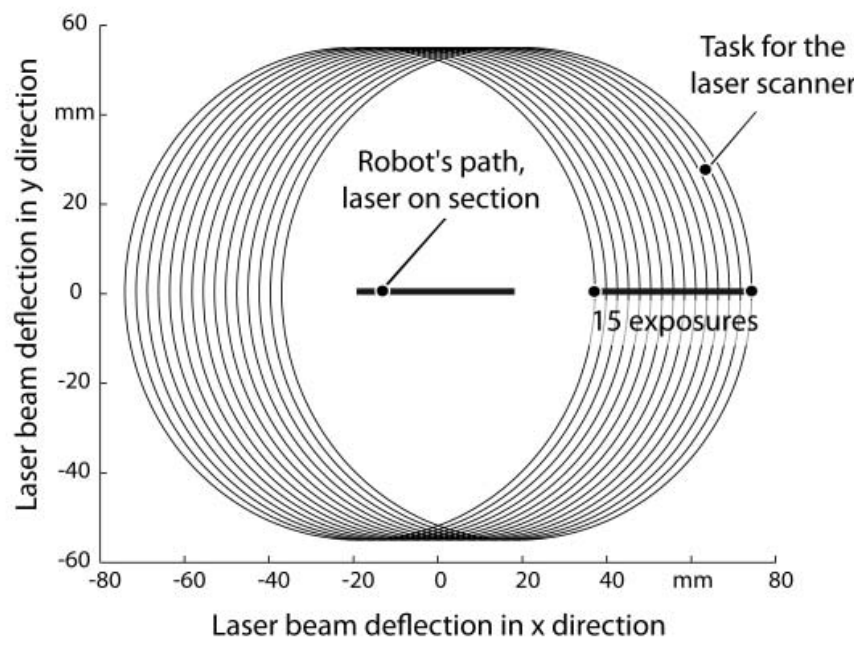

Fig. 9 Resulting path for the laser scanner.

scanner. The advantage of this procedure is the utilization of the standard and proved control systems of the robot and the laser scanner. This makes the system more acceptable in an industrial application compared to solutions which utilize their own custom-built programming interfaces.

In this system setup the robot is considered as the master that starts the laser scanner once the robot reaches the first subtask. Then, both systems run synchronously until one straight line has ended. The robot restarts the laser scanner for each straight line movement. Thereby, the systems stay synchronous during the processing of the whole task.

\section{Applying the Concept to a Heat Exchanger Device}

The described programming method is demonstrated for manufacturing of a heat exchanger device. The programming of the whole system is reduced to teach the center points of the eight cut outs and of the two circular shaped welding seams. The circles were generated using predefined functions, which only need the center and the radius as input parameters. The large welding seam can be converted from CAD data to the task oriented programming using a best fit algorithm [6,9]. Having the task description of the heat exchanger device, the programs for the robot and the laser scanner were generated automatically according to the described concept. After transferring the programs to the corresponding controllers the heat exchanger can be correspondingly machined.

\section{CONCLUSION AND OUTLOOK}

Remote laser beam processing is a promising technology to improve the automation and quality of several manufacturing processes. Reduction of the process cycle time and the complexity of the programming are the main challenges that have to be overcome for an application of these processes. However, the combination of an industrial robot and a laser scanner leads to this conflict. The laser scanner is necessary to shorten the process auxiliary time, but raises the complexity of the whole system.
In this paper a concept was presented for the automated programming of an industrial robot and a laser scanner for remote laser beam cutting, using task oriented programming. The implementation of the concept was demonstrated for a common work piece: a heat exchanger device. Applying this concept the advantages of the robot and the laser scanner are combined to process the work piece.

This system can be used for subtasks that are smaller than the working field of the laser scanner, yet. If it exceeds the working field, two different strategies can be applied. At RLW a combined motion of the robot and the scanner can be realized $[3,6]$. At RLC the robot is too slow. Therefore, the cutting kerf has to be divided into several cutting kerfs that fit into the working field.

Up to now laser beam sources that can be used for RLC are still very expensive and programming systems for RLC with an on-the-fly motion have been tested in laboratory environments, only. To apply RLC into the manufacturing the described concept should be implemented into a fully integrated system controlling the robot and the laser scanner. This is necessary to meet the safety requirements of industrial manufacturing.

\section{ACKNOWLEDGMENT}

The results presented in this paper were gathered within the research project RoboLaSS, Roboter geführter Laser zum Schweißen und Schneiden (Robot guided laser for welding and cutting), funded by the German Federal Ministry of Education and Research (BMBF) and attended by Verein Deutscher Ingenieure e.V. (VDI).

\section{REFERENCES}

[1] D. Havrilla, T. H. Ryba, "High Power Disk Lasers - Advances \& Applications", Congress Proceedings of the $30^{\text {th }}$ ICALEO, Orlando, vol. 104, pp. 749-755, 2011.

[2] J. Musiol, M. Luetke, M. Schweier, J. Hatwig, A. Wetzig, E. Beyer, M. F. Zaeh, "Combining remote ablation cutting and remote welding: opportunities and application areas ", Proceedings of SPIE, vol. 8239, San Francisco, January 2012.

[3] W. Becker, M. Beck, R. Bernhardt, "Potentials of Robot-Guided Remote Laser Welding", Laser assisted net shape engineering 4. Bamberg: Meisenbach, pp. 201-210, 2004.

[4] M. F. Zaeh, U. Munzert, F. Oefele, "Robot based Remote-Laser-Welding without Scanner Optics", Proceedings of the Fourth International WLTConference on Lasers in Manufacturing, Munich, AT-Fachverlag, pp. 429-436, 2007.

[5] C. Thomy, M. Grupp, T. Seefeld, G. Sepold, F. Vollertsen, "Remote Welding of steel and aluminium alloys at high laser power", Laser assisted net shape engineering 4. Bamberg: Meisenbach, pp. 211-222, 2004.

[6] W. Vogl, "Roboterbasiertes Scannerschweißen: effizient und flexibel", EALA - European Automotive Laser Applications, Bad Nauheim, February 2012.

[7] M. Lütke, T. Himmer, A. Wetzig, E. Beyer, "Opportunities to Enlarge the Application Area of Remote-Cutting", Congress Proceedings of the $28^{\text {th }}$ ICALEO, Orlando, vol. 102, pp. 311-318, 2009.

[8] G. Reinhart, M. F. Zaeh, J. Hatwig, "Automated Task Planning for Industrial Robots and Laser Scanners for Remote Laser Beam Welding and Cutting", Production Engineering (2010) 4, pp. 327-332, 2010.

[9] A. Watanabe, S. Sakakibara, K. Ban, M. Yamada, G. Shen, "Autonomous Visual Measurement for Accurate Setting of Workpieces in Robotic Cells", CIRP Annals - Manufacturing Technology, vol. 54, no. 1, pp. 13$18,2005$. 